\title{
Prognostic significance of lymph node ratio after neoadjuvant chemoradiation therapy for esophageal squamous cell
}

\section{carcinoma}

\author{
Jeong Yun Jang ${ }^{1}$, Jesang Yu ${ }^{1}$, Kye Jin Song ${ }^{1}$, Yoon young Jo ${ }^{1}$, Ye Jin Yoo ${ }^{1}$, Sung-Bae Kim², Sook Ryun Park ${ }^{2}$, \\ Young-Hee Kim ${ }^{3}$, Hyeong Ryul Kim³ ${ }^{3}$ Jong Hoon Kim \\ ${ }^{1}$ Department of Radiation Oncology, Asan Medical Center, University of Ulsan College of Medicine, Seoul, Korea \\ ${ }^{2}$ Department of Medical Oncology, Asan Medical Center, University of Ulsan College of Medicine, Seoul, Korea \\ ${ }^{3}$ Department of Thoracic and Cardiovascular Surgery, Asan Medical Center, University of Ulsan College of Medicine, Seoul, Korea
}

Received: October 16, 2020

Revised: November 17, 2020

Accepted: November 18, 2020

Correspondence:

Jong Hoon Kim

Department of Radiation Oncology,

Asan Medical Center, University of

Ulsan College of Medicine, 88

Olympic-ro 43-gil, Songpa-gu, Seoul

05505, Korea.

Tel: +82-2-3010-5680

Fax: +82-2-3010-6950

E-mail: jhkim2@amc.seoul.kr

ORCID:

https://orcid.org/0000-0001-9002-1195
Purpose: We retrospectively evaluated the prognostic significance of lymph node ratio (LNR) in patients with esophageal squamous cell carcinoma who underwent neoadjuvant concurrent chemoradiation therapy (NCRT) followed by surgery.

Materials and Methods: In total, 270 patients who underwent NCRT followed by surgery between August 2005 and December 2015 were included. They were divided into three groups: LNR 0 ( $\mathrm{n}=$ 196), LNR low $(0<L N R \leq 0.1 ; n=63)$, and LNR high $(>0.1 ; n=11)$. The primary endpoint was overall survival (OS), and the secondary endpoints were freedom from local recurrence (FFLR), distant metastasis-free survival (DMFS), and disease-free survival (DFS).

Results: The median number of retrieved lymph nodes per patient was 33. Pathologically, 74 patients had positive lymph nodes. The median follow-up duration was 36.1 months, and the median survival period was 68.4 months. There was a significant correlation between LNR and the number of positive lymph nodes (correlation coefficient $=0.763, p<0.001$ ). There was a substantial difference in the OS among the LNR groups, with 2-year survival rates of 79.0\%,54.0\%, and 9.1\% in the LNR 0, LNR low, and LNR high groups, respectively $(p<0.001)$. A marked decrease in FFLP, DMFS, and DFS was observed with the increasing LNR. In subgroup analysis, the survival results of patients with clinically positive lymph node were similar from those of entire cohort.

Conclusion: LNR is a significant prognostic factor in patients with esophageal squamous cell carcinoma who underwent NCRT followed by surgery. Additional treatment and closer follow-up would be necessary for patients with a high LNR.

Keywords: Esophageal squamous cell carcinoma, Radiotherapy, Lymph node ratio, Prognosis

\section{Introduction}

Esophageal cancer has the seventh highest incidence rate and 6th highest mortality rate among all type of cancer worldwide, accounting for 1 of 20 cancer deaths in 2018 [1]. Despite advancement in the staging system and treatment techniques for esophageal cancer such as surgery, chemotherapy, and radiation therapy, its overall survival (OS) rate is low [2].

Among various prognostic factors, not only the presence of lymph node metastasis, but also the number of lymph node metastases is important, and its significance was reflected in staging systems of the American Joint Committee on Cancer (AJCC) and the Union for International Cancer Control (UICC) in 2009 [3-5]. In addition, many studies have reported that the number of lymph 
nodes removed during surgery was a significant factor affecting the prognosis of patients $[6,7]$. Hence, the concept of lymph node ratio (LNR), the ratio of the number of positive lymph nodes to the total number of removed lymph nodes, has gained attention as an important prognostic factor in gastrointestinal cancers including esophageal cancer [8-13]. However, studies on LNR in squamous cell carcinoma $(\mathrm{SqCC})$ are relatively rare, as the majority of the studies were conducted in Western countries where adenocarcinoma is the dominant histology. In addition, these studies have limitations in the heterogeneity of treatment methods and absence of a subgroup analysis. Hence, it may not be appropriate to apply LNR reported in previous studies to the current clinical conditions.

Therefore, this study aimed to investigate the importance of LNR as a prognostic factor in patients with esophageal squamous cell carcinoma who underwent neoadjuvant concurrent chemoradiation therapy (NCRT) followed by surgery.

\section{Materials and Methods}

\section{Patients}

We retrospectively reviewed the medical records of patients who underwent NCRT at Asan Medical Center between August 2005 and December 2015. All patients underwent surgery after NCRT, and the pathological results were confirmed. The exclusion criteria were as follows: patients with double primary cancer within 5 years before the diagnosis of esophageal cancer, those with histology other than $\mathrm{SqCC}$, those with distant metastases at the time of diagnosis, those receiving a radiation dose of $<36 \mathrm{~Gy}$, and those with a short follow-up period after surgery ( $<3$ months). In total, 270 patients were enrolled in this study; all patients had undergone esophagogastroduodenoscopy (EGD) with biopsy, endoscopic ultrasonography (EUS), chest computed tomography (CT), and positron emission tomography-computed tomography (PET-CT) for appropriate staging. The informed consent was waived.

\section{Treatments and follow-up}

All patients were administered XP, FP, or oxaliplatin/TS-1 chemotherapy as NCRT. In the XP regimen, patients received capecitabine $1,600 \mathrm{mg} / \mathrm{m}^{2} /$ day for 5 days plus cisplatin $30 \mathrm{mg} / \mathrm{m}^{2} /$ day on the first day, weekly. In the FP regimen, patients received cisplatin 60 $\mathrm{mg} / \mathrm{m}^{2} /$ day on the first day plus 5 -fluorouracil $1,000 \mathrm{mg} / \mathrm{m}^{2} /$ day on the second day for 4 days, every 3 weeks. In the oxaliplatin/TS-1 regimen, patients received TS- $150 \mathrm{mg}$ orally twice a day daily during the whole period of concurrent chemoradiation therapy, and oxaliplatin $130 \mathrm{mg} / \mathrm{m}^{2}$ as an intravenous infusion on day 1, repeated every 3 weeks. The median dose of radiation therapy was $46 \mathrm{~Gy}$ (range, 38 to $50.4 \mathrm{~Gy}$ ), and the fraction size was either $180 \mathrm{cGy}$ or
200 cGy. Since the aim of radiotherapy was neoadjuvant treatment, elective nodal irradiation was used in most cases, and the range of field was customized according to the locations of the primary lesion and metastatic lymph nodes. EGD, chest CT, and PET-CT were used to evaluate tumor response and absence of new metastasis at 1 month after NCRT before proceeding with surgery. All surgeries were performed by expert surgeons with experience in performing $>50$ radical esophagectomies per year. The median interval between the last day of NCRT and surgery was 8 weeks. In total, $65.9 \%$ patients underwent Ivor-Lewis operation and 34.1\% patients underwent McKeown operation, and a sufficient number of lymph nodes were retrieved to evaluate the post-treatment pathological stage according to the AJCC 7th edition [14]. We derived LNR and classified patients into three groups: LNR 0, LNR low $(0<$ LNR $\leq 0.1)$, and LNR high (>0.1).

All patients were regularly followed up by a radiation oncologist, medical oncologist, and thoracic surgeon. At each follow-up, history taking, physical examination, and blood tests (complete blood count and blood biochemistry tests) were performed. Chest CT and abdominal and pelvic $\mathrm{CT}$, if needed, were performed every 6 months for 2 years and annually for 5 years. EGD was performed every year, and biopsy was performed selectively only when tumor recurrence was suspected. PET-CT was not mandatory and was performed only when tumor recurrence was suspected.

\section{Outcomes and statistical analysis}

The primary endpoint was OS, and the secondary endpoints were freedom from local recurrence (FFLR), distant metastasis-free survival (DMFS), and disease-free survival (DFS). Statistical analyses were performed using SPSS version 22.0 (IBM SPSS, Armonk, NY, USA). Pearson correlation analysis was performed to determine the correlation between LNR and the number of positive lymph nodes. Univariate analysis was performed through logistic regression analysis, and a Cox proportional hazard model was used for multivariate analysis to find prognostic risk factors. The Kaplan-Meier method was used to derive the survival outcome.

\section{Results}

\section{Patients and treatments}

A total of 270 patients were included, and their characteristics are presented in Table 1. The median age was 63 years (range, 42 to 76 years), and $94.4 \%$ patients were men. Tumors were confirmed by endoscopic biopsy before initiating treatment. All patients were diagnosed with SqCC. Tumor location was categorized into the upper, mid-, and lower thoracic esophagus by measuring the distance from the upper incisor to the tumor epicenter using endoscopic 
Table 1. Patients' characteristics

\begin{tabular}{|c|c|c|c|c|c|}
\hline \multirow{2}{*}{ Characteristic } & \multicolumn{4}{|c|}{ LNR } & \multirow{2}{*}{ p-value } \\
\hline & $0(n=196)$ & Low $(n=63)$ & High $(n=11)$ & Total $(n=270)$ & \\
\hline Age (yr) & $63(42-76)$ & $59(52-73)$ & $159(56-75)$ & $63(42-76)$ & 0.059 \\
\hline Sex & & & & & 0.696 \\
\hline Male & $185(94.4)$ & 59 (93.6) & $11(100)$ & $255(94.4)$ & \\
\hline Female & $11(5.6)$ & $4(6.4)$ & $0(0)$ & $15(5.6)$ & \\
\hline Tumor differentiation & & & & & 0.231 \\
\hline Well & $30(15.3)$ & $2(3.2)$ & $2(18.2)$ & 34 (12.6) & \\
\hline Moderately & $143(73.0)$ & $7(11.1)$ & $8(72.7)$ & $204(75.6)$ & \\
\hline Poor & $16(8.2)$ & $53(84.1)$ & $1(9.1)$ & $24(8.9)$ & \\
\hline Not checkable & $7(3.6)$ & $1(1.6)$ & $0(0)$ & $8(3.0)$ & \\
\hline Tumor location ${ }^{\text {a) }}$ & & & & & 0.449 \\
\hline Upper & 24 (12.2) & 10 (15.9) & $1(9.1)$ & 35 (13.0) & \\
\hline Middle & $100(51.0)$ & $22(35.0)$ & $6(54.5)$ & $128(47.4)$ & \\
\hline Lower & $72(36.7)$ & $31(49.2)$ & $4(36.4)$ & $107(39.6)$ & \\
\hline Initial clinical stage & & & & & 0.033 \\
\hline I & $17(8.7)$ & $8(12.7)$ & $1(0.9)$ & $26(9.6)$ & \\
\hline ॥ & $57(29.1)$ & $17(27.0)$ & $0(0)$ & $74(27.4)$ & \\
\hline III & $117(59.7)$ & $31(49.2)$ & $9(81.8)$ & $157(58.1)$ & \\
\hline IV & $4(2.0)$ & 7 (11.1) & $1(0.9)$ & $12(4.4)$ & \\
\hline Clinical T stage & & & & & 0.167 \\
\hline cT1 & $21(10.7)$ & $8(12.7)$ & $1(9.1)$ & $30(11.1)$ & \\
\hline cT2 & $58(29.6)$ & $23(36.5)$ & $1(9.1)$ & $82(30.4)$ & \\
\hline cT3 & $115(58.7)$ & $29(46.0)$ & 9 (81.8) & $153(56.7)$ & \\
\hline cT4 & $2(1.0)$ & $3(4.8)$ & $0(0)$ & $5(1.9)$ & \\
\hline Clinical N stage & & & & & $<0.001$ \\
\hline Positive & $131(66.8)$ & $58(92.1)$ & 9 (81.8) & $198(73.3)$ & \\
\hline Negative & 65 (33.2) & $5(7.9)$ & $2(18.2)$ & $72(26.7)$ & \\
\hline LNs examined & $33(8-69)$ & $36(13-67)$ & $21(6-33)$ & $33(6-69)$ & 0.582 \\
\hline LNs positive & $0(0-0)$ & $1(1-4)$ & $4(2-8)$ & $0(0-8)$ & $<0.001$ \\
\hline \multicolumn{6}{|l|}{ Chemotherapy } \\
\hline$X P$ & 152 (77.6) & 48 (76.2) & $10(90.9)$ & $210(77.8)$ & 0.668 \\
\hline FP & $3(1.5)$ & $0(0)$ & $0(0)$ & $3(1.1)$ & \\
\hline 0xaliplatin/TS-1 & $41(20.9)$ & $15(23.8)$ & $1(9.1)$ & $57(21.1)$ & \\
\hline Operation & & & & & 0.932 \\
\hline Ivor-Lewis operation & $131(66.8)$ & $40(63.5)$ & $7(63.6)$ & $178(65.9)$ & \\
\hline McKeown operation & 65 (33.2) & $23(36.5)$ & $4(36.4)$ & $92(34.1)$ & \\
\hline
\end{tabular}

Values are presented as median (range) or number (\%). Because of rounding, not all percentages total 100.

LNR, lymph node ratio; LN, lymph node; XP, capecitabine/cisplatin; FP, 5-fluorouracil/cisplatin.

${ }^{\text {a) }}$ Classification by measuring the distance from the upper incisor (UI) to the tumor by esophagogastroscopy (upper, UI $20-25 \mathrm{~cm}$; mid, UI 25-30 cm; and lower, $30-40 \mathrm{~cm}$ ).

evaluation and other imaging studies. Approximately half of the patients had lesions in the mid-thoracic esophagus, and upper thoracic esophageal cancer was the least. Most patients had advanced stage disease, for which NCRT and surgery were recommended as the standard treatment [15]. However, some patients had early-stage disease. Clinically suspected lymph nodes were found in 73.3\% patients.

\section{Correlation between LNR and the number of positive lymph node}

In 270 patients, a total of 9,150 lymph nodes were removed during surgery, of which 128 (1.4\%) were identified as metastatic nodes. The median number of lymph nodes harvested per person during surgery was 33 (range, 8 to 69). Pearson correlation analysis showed that there was a significant correlation between LNR and the number of metastatic lymph nodes (correlation coefficient = 
0.763, p<0.001) (Fig. 1).

\section{OS, LCR, DFS, and DMFS according to LNR}

The median follow-up period was 36.1 months (range, 5.3 to 164 months). In the entire study cohort, the median OS time was 68.4 months (range, 5.3 to 172.2 months) and the 2- and 5-year OS rate were $70.2 \%$ and $52.4 \%$, respectively. Table 2 shows the survival outcomes in all study groups. The median survival period according to LNR was 81.2 months in the LNR 0 group, 35.6 months in the LNR low group, and 11 months in the LNR high group ( $<0.001$ ). The 2- and 5-year survival rates in the LNR 0, low, and high groups were $79.0 \%, 54.0 \%$, and $9.1 \%$ and $60.2 \%, 37.6 \%$, and $0.0 \%$, re-

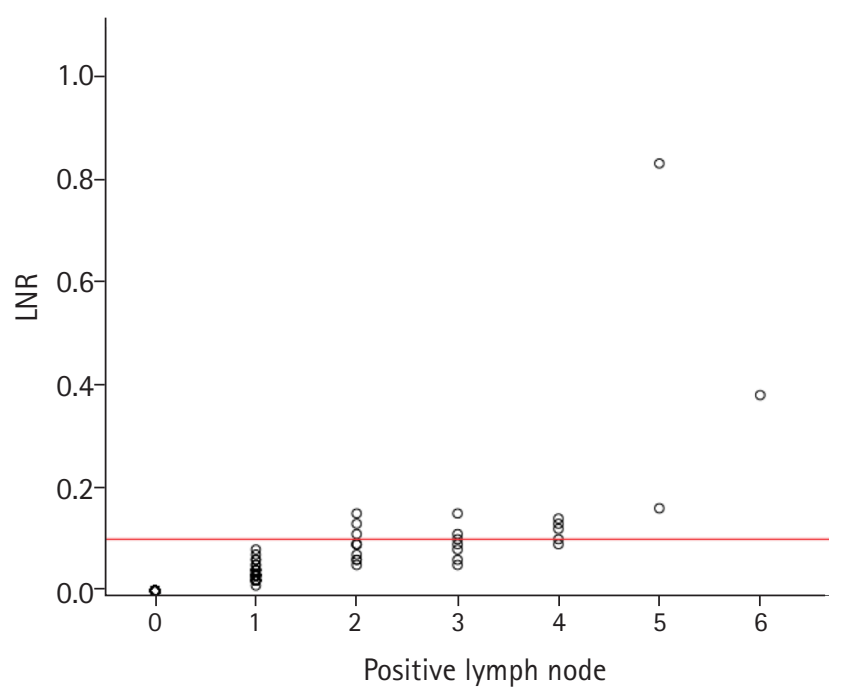

Fig. 1. Correlation between lymph node ratio (LNR) and the number of positive lymph node. spectively $(p<0.001)$ (Fig. 2A). Four variables (initial clinical stage, clinical nodal stage, pathologic nodal stage, and LNR) had prognostic significance on Cox regression analysis for OS (Table 3).

Local recurrence occurred in 59 patients (21.9\%), distant metastasis occurred in 49 patients (18.1\%), and both local recurrence and distant metastasis occurred in 29 patients (10.7\%). Local recurrence rates were $16.3 \%, 33.3 \%$, and $54.5 \%$ in the LNR 0, LNR low, and LNR high groups, respectively.

The 5-year FFLR, DMFS, and DFS rates in the entire study cohort were $76.0 \%, 78.5 \%$, and 68.1\%, respectively. The 5-year FFLR, DMFS, and DFS rates were significantly different among all three LNR groups, respectively; the smaller the LNR value, the better the survival rate (Fig. 2B-2D).

The 2-year DMFS rates in the LNR 0, low, and high groups were $91.6 \%, 78.1 \%$, and $0.0 \%$, respectively, showing significant differences among the groups. The difference was more prominent between the LNR high group and the other groups $(p<0.001)$ than that between the LNR 0 and LNR low groups ( $p=0.044$ ) (Fig. 2C). Of the 11 patients with high LNR, 3 patients had lung metastasis, 1 patient had liver metastasis, and 1 patient had multiple lymph node metastases.

The same analyses were performed in the subgroup of patients in whom positive lymph node was diagnosed on EUS or CT before NCRT. The results of subgroup were similar from those of entire cohort (Table 4). There were 198 patients (73.3\%) with clinical node positive, of which 126 patients expired. Median OS was 48.3 months, about 20 months shorter compared to the OS for the entire cohort. Their median OS was 75.1 months, 34.8 months, and 10.3 months for LNR 0, low, and high, respectively, and a lower

Table 2. The survival outcomes according to LNR

\begin{tabular}{|c|c|c|c|c|c|c|}
\hline & \multirow{3}{*}{ LNR } & \multicolumn{3}{|c|}{ Median survival time (mo) } & \multirow{3}{*}{2 -yr survival rate $(\%)$} & \multirow{3}{*}{ p-value } \\
\hline & & \multirow{2}{*}{ Kaplan-Meier estimate } & \multicolumn{2}{|c|}{ 95\% confidence limits } & & \\
\hline & & & Lower & Upper & & \\
\hline \multirow[t]{3}{*}{ OS } & 0 & 81.2 & 51.6 & 110.8 & 79.0 & $<0.001$ \\
\hline & Low & 35.6 & 14.8 & 56.4 & 54.0 & \\
\hline & High & 11.0 & 6.9 & 15.1 & 9.1 & \\
\hline \multirow[t]{3}{*}{ FFLR } & 0 & $N / R$ & - & - & 85.9 & $<0.001$ \\
\hline & Low & $N / R$ & - & - & 69.7 & \\
\hline & High & 13.2 & 8.4 & 18 & 0.0 & \\
\hline \multirow[t]{3}{*}{ DMFS } & 0 & $N / R$ & - & - & 91.6 & $<0.001$ \\
\hline & Low & $N / R$ & - & - & 78.1 & \\
\hline & High & 17.1 & 4.5 & 29.7 & 0.0 & \\
\hline \multirow[t]{3}{*}{ DFS } & 0 & $N / R$ & - & - & 80.1 & $<0.001$ \\
\hline & Low & $N / R$ & - & - & 61.2 & \\
\hline & High & 8.6 & 3.7 & 13.5 & 0.0 & \\
\hline
\end{tabular}

OS, overall survival; FFLR, free from local recur; DMFS, distant metastasis-free survival; DFS, disease-free survival; LNR, lymph node ratio; N/R, not reached. 

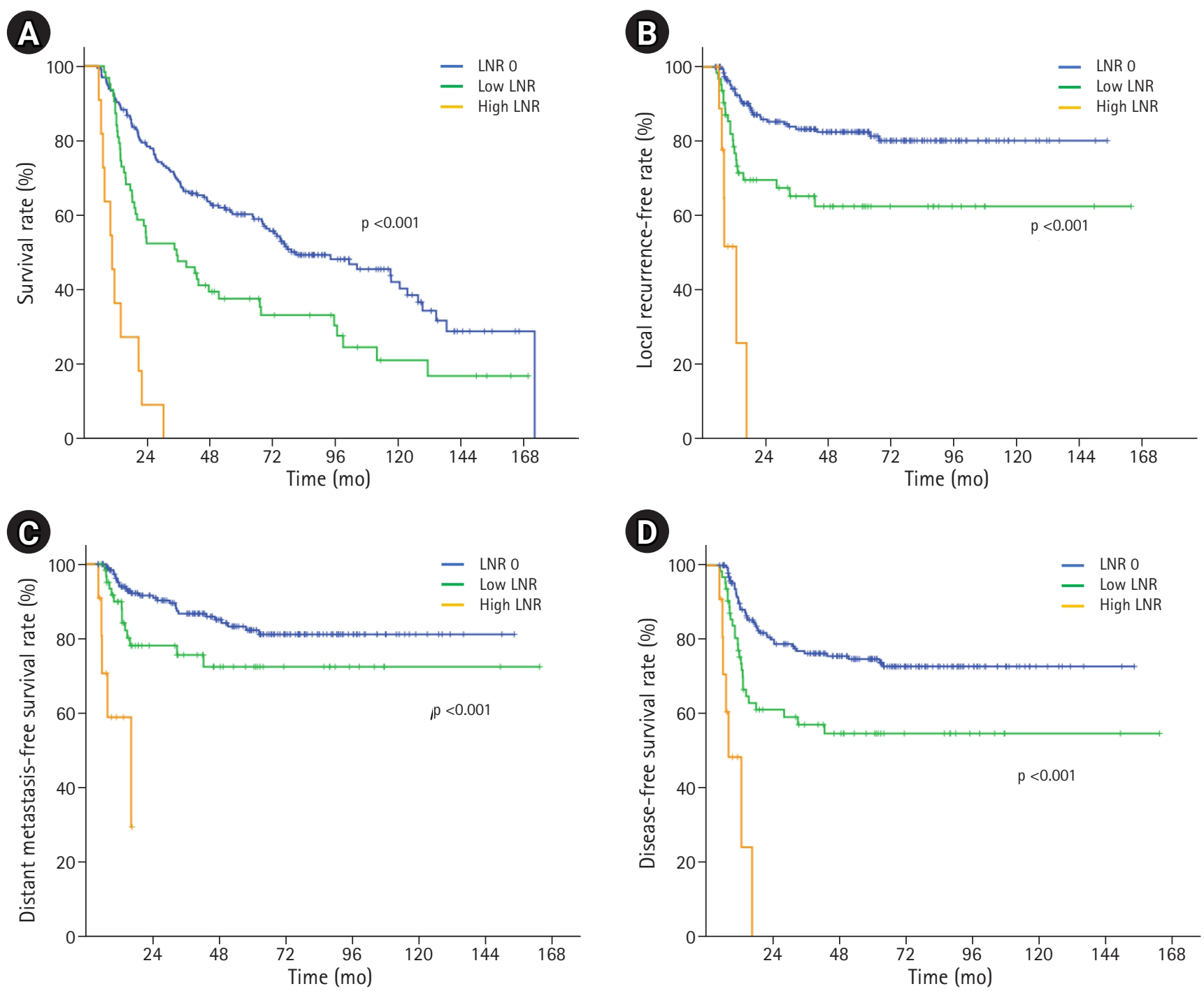

Fig. 2. The survival outcomes according to lymph node ratio (LNR): (A) overall survival rate, (B) local recurrence-free rate, (C) distant metastasis-free survival rate, and (D) disease-free survival rate.

survival rate was shown in the group with a large LNR value, as in all patients ( $p$ 0.001). In addition, FFLR, DMFS, and DFS values also showed significant differences among all three LNR groups, confirming a low survival rate in the high LNR group.

As another subgroup analysis, patients were divided by pathologic N stages. In ypNO patients, we tried to determine the difference in survival according to clinical nodal status, and in patients with positive pathologic lymph node, we tried to find out the difference according to the LNR values. First, there were a total of 196 patients with ypN0, of which 65 and 131 patients were clinical node negative and positive, respectively. The 2-year OS rate was statistically higher in patients with negative clinical nodal status with $89.1 \%(p=0.017)$. However, there were no significant differences in FFLR, DMFS, and DFS between two groups (Supplementary Table S1). Patients with positive pathologic nodal status were clas- sified into ypN1, N2, and N3, and the numbers were 59, 14, and 1, respectively. Due to the small number of patients in ypN3 stage, the analysis was performed in two groups, ypN1 and ypN2-3. In ypN1, patients with low LNR showed better survival in all except DMFS. The median OS for high LNR and low LNR showed a difference of about five times at 14.3 months and 69.3 months $(p=0.008)$. However, in the ypN2-3 group, there was no significant difference between the low LNR and the high LNR patients in any survival outcome (Supplementary Table S2).

\section{Discussion and Conclusion}

In this study, we found that LNR is a significant prognostic factor associated with OS. It is difficult to directly compare most previous studies with the current study because they included patients who 
Table 3. Univariate and multivariate analysis for overall survival $(n=270)$

\begin{tabular}{|c|c|c|c|c|c|c|}
\hline \multirow{2}{*}{ Variable } & \multirow{2}{*}{ No. of patients } & \multicolumn{2}{|c|}{ Univariate analysis } & \multicolumn{3}{|c|}{ Multivariate analysis } \\
\hline & & 2 -yr survival rate $(\%)$ & $p$-value & HR & $95 \% \mathrm{Cl}$ & $p$-value \\
\hline Age (yr) & & & 0.167 & & & \\
\hline$\leq 63$ & 164 & 68.9 & & & & \\
\hline$>63$ & 106 & 72.3 & & & & \\
\hline Sex & & & 0.338 & & & \\
\hline Male & 255 & 70.4 & & & & \\
\hline Female & 15 & 66.7 & & & & \\
\hline Tumor differentiation & & & 0.597 & & & \\
\hline Well & 24 & 82.4 & & & & \\
\hline Moderately & 204 & 66.5 & & & & \\
\hline Poor & 24 & 83.3 & & & & \\
\hline Not checkable & 8 & 75.0 & & & & \\
\hline Tumor location ${ }^{\text {a) }}$ & & & 0.933 & & & \\
\hline Upper & 34 & 70.6 & & & & \\
\hline Middle & 128 & 70.3 & & & & \\
\hline Lower & 108 & 69.8 & & & & \\
\hline Initial clinical stage & & & 0.001 & & & 0.011 \\
\hline I & 26 & 92.3 & & 1 & Referent & \\
\hline ॥ & 74 & 78.3 & & 1.739 & $1.206-2.506$ & \\
\hline III & 157 & 62.9 & & 8.15 & $4.203-15.802$ & \\
\hline $\mathrm{IVa}$ & 13 & 69.2 & & 1.599 & $1.067-2.397$ & \\
\hline Clinical nodal stage & & & 0.003 & & & 0.023 \\
\hline Positive & 198 & 65.1 & & 1 & Referent & \\
\hline Negative & 72 & 84.6 & & 1.599 & $10.67-2.397$ & \\
\hline Pathological nodal stage ${ }^{\text {b) }}$ & & & $<0.001$ & & & \\
\hline ypNo & 196 & 79.0 & & & & \\
\hline ypN1 & 59 & 55.9 & & & & \\
\hline ypN2-3 & 15 & 13.3 & & & & \\
\hline LNs examined & & & 0.726 & & & \\
\hline$\leq 15$ & 13 & 53.8 & & & & \\
\hline$>15$ & 257 & 71.1 & & & & \\
\hline LNR & & & $<0.001$ & & & $<0.001$ \\
\hline 0 & 196 & 79.0 & & 1 & Referent & \\
\hline Low $(>0$ and $\leq 0.1)$ & 63 & 54.0 & & 1.739 & $1.206-2.506$ & \\
\hline High (>0.1) & 11 & 9.1 & & 8.15 & $4.203-15.802$ & \\
\hline Chemotherapy & & & 0.572 & & & \\
\hline$X P$ & 210 & 69.4 & & & & \\
\hline $\mathrm{FP}$ & 3 & 66.7 & & & & \\
\hline 0xaliplatin/TS-1 & 57 & 76.9 & & & & \\
\hline Operation & & & 0.827 & & & \\
\hline Ivor-Lewis operation & 178 & 69.4 & & & & \\
\hline McKeown operation & 92 & 73.3 & & & & \\
\hline
\end{tabular}

$\mathrm{HR}$, hazard ratio; $\mathrm{Cl}$, confidence interval; LNR, lymph node ratio; LN, lymph node; XP, capecitabine/cisplatin; FP, 5-fluorouracil/cisplatin.

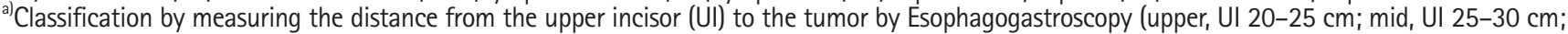
lower, 30-40 cm).

${ }^{b)}$ Due to strong linear relationship between the pathologic $\mathrm{N}$ stage and LNR, only LNR was included in the multivariate analysis. 
Table 4. The survival outcomes according to LNR for patients with clinically positive lymph node

\begin{tabular}{|c|c|c|c|c|c|c|}
\hline & \multirow{3}{*}{ LNR } & \multicolumn{3}{|c|}{ Median survival time (mo) } & \multirow{3}{*}{2 -yr survival rate $(\%)$} & \multirow{3}{*}{$\mathrm{p}$-value } \\
\hline & & \multirow{2}{*}{ Kaplan-Meier estimate } & \multicolumn{2}{|c|}{ 95\% confidence limits } & & \\
\hline & & & Lower & Upper & & \\
\hline \multirow[t]{3}{*}{ OS } & 0 & 75.1 & 62.9 & 87.3 & 74.0 & $<0.001$ \\
\hline & Low & 34.8 & 15.5 & 54.1 & 53.4 & \\
\hline & High & 10.3 & 3.9 & 16.7 & 11.1 & \\
\hline \multirow[t]{3}{*}{ FFLR } & 0 & $N / R$ & - & - & 82.1 & $<0.001$ \\
\hline & Low & $N / R$ & - & - & 67.3 & \\
\hline & High & 8.6 & 7.6 & 9.6 & 0.0 & \\
\hline \multirow[t]{3}{*}{ DMFS } & 0 & $N / R$ & - & - & 89.2 & 0.001 \\
\hline & Low & $N / R$ & - & - & 79.9 & \\
\hline & High & 8.6 & - & - & 47.6 & \\
\hline \multirow[t]{3}{*}{ DFS } & 0 & $N / R$ & - & - & 75.9 & $<0.001$ \\
\hline & Low & $N / R$ & - & - & 61.4 & \\
\hline & High & 8.6 & 6.2 & 11.0 & 0.0 & \\
\hline
\end{tabular}

OS, overall survival; FFLR, free from local recur; DMFS, distant metastasis-free survival; DFS, disease-free survival; LNR, lymph node ratio; N/R, not reached.

received inhomogeneous treatment (NCRT followed by surgery and surgery alone cases together), had inhomogeneous histology (both adenocarcinoma and SqCC together), or had inhomogeneous stages. For example, in the study conducted by Nigro et al. [10], all patients had adenocarcinoma and they were assigned only to the surgery group. In the study by Bollschweiler et al. [3], approximately half of the patients had SqCC and the other patients had adenocarcinoma; 62\% patients were included the surgery alone group. However, the study by Wilson et al. [12] included a more homogeneous patient group in which $>80 \%$ patients had SqCC and most patients underwent NCRT. However, the median OS was 22 months, and the 5-year survival rate was $27 \%$, which was slightly lower than the expected values. Compared with the study by Liu et al. [13] that enrolled SqCC in near 100\%, their median OS was 36.7 months, which was very similar to that of 36.1 months in our study. From the above studies, we could draw a very reasonable conclusion that there is a significant correlation between LNR and surviv$\mathrm{al}$, and the higher the LNR, the lower the survival rate.

In our study, the distribution of patient characteristics in each group was quite even, except initial clinical stage and clinical node status; however, uneven distribution of clinically positive lymph nodes could have affected the survival outcome. Hence, we performed subgroup analysis in patients with clinically positive lymph node. In this subgroup, we could confirm that not only the OS but also the tendency of local recurrence and distant metastasis were repeated, which is the same as the results of entire cohort patients. Therefore, we can conclude that LNR is a significant prognostic factor regardless of the clinical nodal status.

In addition, we have questioned how much LNR means when only patients within the same pathologic stage are studied. Therefore, another subgroup analysis was performed for patients with ypN1 and ypN2-3 by dividing them into low LNR and high LNR groups. In the ypN1 group, low LNR patients showed better outcomes in OS, FFLR, and DFS. Through this, we found that the survival difference was shown according to the LNR value even within the same pathologic stage. Consequently, not only positive lymph node but also LNR with the consideration of the number of removed lymph node could provide additional information in predicting the patient's prognosis. However, since the number of each LNR subgroup is not sufficient, an analysis including a larger number of patients to confirm the results of this study is necessary. On the other hand, in patients with ypN2-3, there was no significant difference in survival outcomes according to the LNR value. It can be interpreted that LNR does not act as a prognostic factor within this stage, but this result may have been derived because the number of patients is extremely small in both high LNR and low LNR groups. Therefore, it will be difficult to assert that LNR has no meaning in patients with high pathologic $\mathrm{N}$ stage based on this result alone. Therefore, we expect that this also could have resulted in significant results if the study had enough patients.

Of course, as can be seen through the univariate analysis of this study, the clinical and pathologic stage established by AJCC are one of the major risk factors that have a very large influence on the prognosis of patients. Since LNR is the proportion of the number of positive lymph node to the number of retrieved lymph node, it can be readily deduced that it will correlate with the number of metastatic lymph node, which is a well-known risk factor. However, the number of dissected lymph node, which is the denominator, is also 
a key element in the formula for calculating the LNR, so it would be difficult to say that there is always a correlation between two variables. Therefore, an analysis was conducted, and a distinct positive correlation was proved. Despite this result, when univariate and multivariate analyses were performed, LNR itself was found to be a prognostic factor for survival. For this reason, in addition to clinical and pathological stages, it will also be necessary to focus on the LNR value, which is emerging as another prognostic factor. Consequently, patients with high LNR values need more careful observation, and early detection of recurrence followed by salvage treatment is more needed than in the other patients.

Another important issue is setting the criteria for "high LNR" and we do not have enough data to make a consensus on that specific value. Many studies have used different cutoff values such as 0.1, $0.2,0.25$, or 0.5 . In the study by Bollschweiler et al. [3], there was a significant difference in survival when the LNR value was $>0.2$ ( $p$ $<0.01$ ), and in the study by Nigro et al. [10], there was a significant difference in survival when the LNR value was 0.1. A literature review revealed that the LNR value that determines significance decreases with the increasing number of the total retrieved lymph node $[3,10,12]$. In this study, the median number of lymph nodes harvested per person was 33, and we set the cutoff value as low as 0.1. Therefore, LNR was divided into three groups according to this cutoff value, and a substantial proportion of patients belonged to the LNR 0 group due to the high ypCR rate in patients with SqCC. Initially, we enrolled 198 patients clinically positive lymph nodes at the time of diagnosis, and 131 of them showed complete response pathologically in nodal status after NCRT, accounting for $66 \%$ of the total ypNO rate. Therefore, recruiting enough number of patients with high LNR in a single institution was challenging and we would like to say it requires multi-institutional cooperation. In this context, we agree that there was a limitation to determine a cutoff value based only on this study, even if we found significant differences in the survival rate based on the LNR value of 0.1 .

Despite this limitation, we believe this study is meaningful as we recruited patients treated with the current standard treatment. Our study is not the first to show the importance of LNR as a prognostic factor, but we can say our results are based on homogeneous data from NCRT and surgery. Second, all of our patients were diagnosed as SqCC, which is dominant in Asian countries and its nature is different from adenocarcinoma. Third, consistency in treatment and follow-up was ensured by the multidisciplinary approach team consensus.

We believe that our results provided data regarding the current clinical situation, and hope it will be a basis for future prospective studies including a large number of patients and considering additional treatment for patients with high LNR.

\section{Conflict of Interest}

No potential conflict of interest was reported by the authors.

\section{Supplementary Materials}

Supplementary materials can be found via https://doi.org/10.3857/ roj.2020.00850.

\section{References}

1. Bray F, Ferlay J, Soerjomataram I, Siegel RL, Torre LA, Jemal A. Global cancer statistics 2018: GLOBOCAN estimates of incidence and mortality worldwide for 36 cancers in 185 countries. CA Cancer J Clin 2018;68:394-424.

2. Egyud MR, Tseng JF, Suzuki K. Multidisciplinary therapy of esophageal cancer. Surg Clin North Am 2019;99:419-37.

3. Bollschweiler E, Baldus SE, Schroder W, Schneider PM, Holscher $\mathrm{AH}$. Staging of esophageal carcinoma: length of tumor and number of involved regional lymph nodes. Are these independent prognostic factors? J Surg Oncol 2006;94:355-63.

4. Hofstetter W, Correa AM, Bekele N, et al. Proposed modification of nodal status in AJCC esophageal cancer staging system. Ann Thorac Surg 2007;84:365-75.

5. Rice TW, Rusch VW, Ishwaran H, Blackstone EH; Worldwide Esophageal Cancer Collaboration. Cancer of the esophagus and esophagogastric junction: data-driven staging for the seventh edition of the American Joint Committee on Cancer/International Union Against Cancer Cancer Staging Manuals. Cancer 2010; 116:3763-73.

6. Peyre CG, Hagen JA, DeMeester SR, et al. The number of lymph nodes removed predicts survival in esophageal cancer: an international study on the impact of extent of surgical resection. Ann Surg 2008;248:549-56.

7. Altorki NK, Zhou XK, Stiles B, et al. Total number of resected lymph nodes predicts survival in esophageal cancer. Ann Surg 2008;248:221-6.

8. Marchet $A_{1}$ Mocellin $S_{1}$ Ambrosi $A$, et al. The ratio between metastatic and examined lymph nodes ( $N$ ratio) is an independent prognostic factor in gastric cancer regardless of the type of lymphadenectomy: results from an Italian multicentric study in 1853 patients. Ann Surg 2007;245:543-52.

9. Berger AC, Sigurdson ER, LeVoyer T, et al. Colon cancer survival is associated with decreasing ratio of metastatic to examined Iymph nodes. J Clin Oncol 2005;23:8706-12.

10. Nigro JJ, DeMeester SR, Hagen JA, et al. Node status in transmural esophageal adenocarcinoma and outcome after en bloc 
esophagectomy. J Thorac Cardiovasc Surg 1999;117:960-8.

11. Mariette C, Piessen G, Briez N, Triboulet JP. The number of metastatic lymph nodes and the ratio between metastatic and examined lymph nodes are independent prognostic factors in esophageal cancer regardless of neoadjuvant chemoradiation or lymphadenectomy extent. Ann Surg 2008;247:365-71.

12. Wilson M, Rosato EL, Chojnacki KA, et al. Prognostic significance of lymph node metastases and ratio in esophageal cancer. J Surg Res 2008;146:11-5.

13. Liu YP, Ma L, Wang SJ, et al. Prognostic value of lymph node me- tastases and lymph node ratio in esophageal squamous cell carcinoma. Eur J Surg Oncol 2010;36:155-9.

14. Edge SB, Compton CC. The American Joint Committee on Cancer: the 7th edition of the AJCC cancer staging manual and the future of TNM. Ann Surg Oncol 2010;17:1471-4.

15. Shapiro J, van Lanschot JJ, Hulshof MC, et al. Neoadjuvant chemoradiotherapy plus surgery versus surgery alone for oesophageal or junctional cancer (CROSS): long-term results of a randomised controlled trial. Lancet Oncol 2015;16:1090-8. 